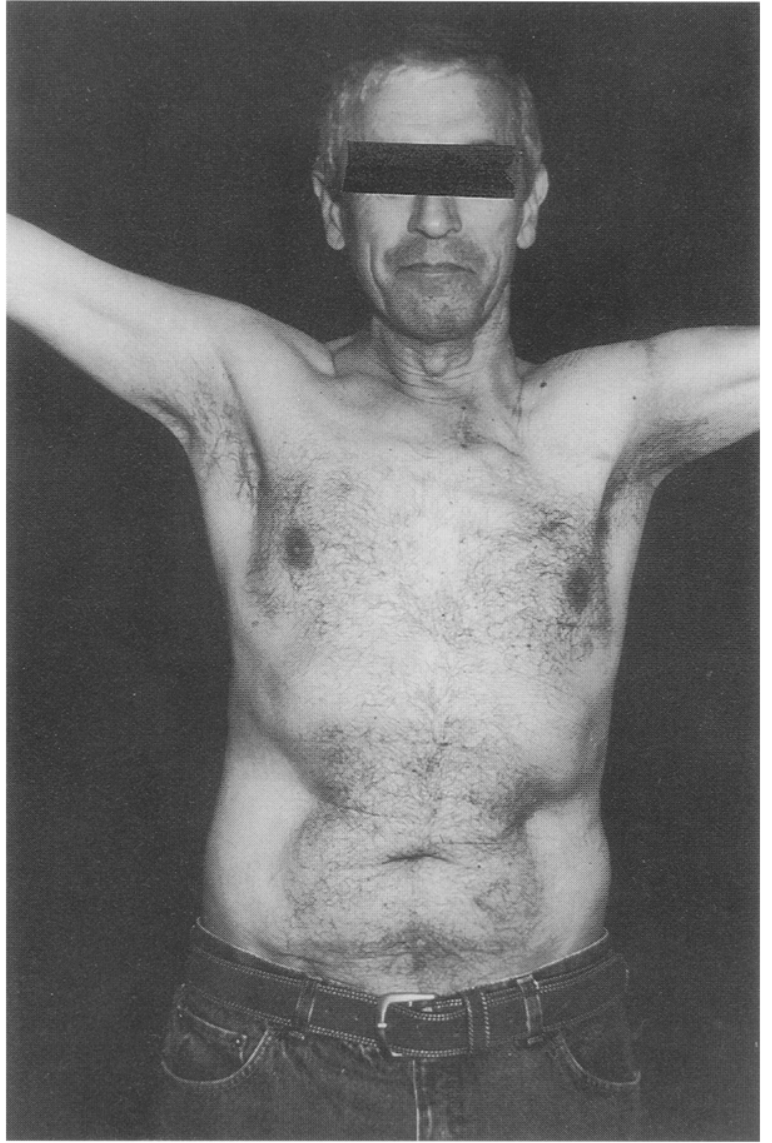

Fig. 1. A 52-year-old man operated on 24 months ago by an anterior transcervical and median posterior approach for a left bronchogenic tumor invading the intervertebral foramen. Note the perfect shoulder mobility even after resection of the clavicle and spinal fixation.

nerve, because this and not the resection of the clavicle by itself causes winging of the scapula.

3. As to whether sparing the clavicle is worthwhile, our surgical philosophy concerning this subject (including its mode of reconstruction) was extensively detailed in the August 1996 issue of this Journal. Perseverare diabolicum.

4. Concerning the remarks as to whether the resection of the clavicle by itself can result in a cosmetic and functional compromise, we enclose a photograph of a 52-year-old patient whose bronchogenic tumor invaded the intervertebral foramen; he had a hemivertebrectomy (T1, T2, T3) through a combined anterior transcervical and posterior midline approach and resection of the clavicle without reconstruction. It does not appear to him or to us that he is cosmetically compromised (Fig. 1). This observation was the general rule among our study population.

For these aforementioned reasons, we strongly believe that sparing the clavicle does not reserve that much pourparler.

Philippe Dartevelle, $M D$

Paolo Macchiarini, $M D$

Department of Thoracic and Vascular Surgery and Heart-Lung Transplantation, Hôpital Marie-

Lannelongue (Paris-Sud University)

133, Avenue de la Resistance,

92350 Le Plessis Robinson, France

$12 / 8 / 79872$

\section{Coronary arteries arising from the contralateral aortic sinus: Electron beam computed tomographic demonstration of the initial course of the artery with respect to the aorta and the right ventricular outflow tract \\ To the Editor:}

In the September issue of this Journal, Mousseaux and colleagues ${ }^{1}$ present an elegant demonstration of the efficiency of computed tomography in demonstrating the anomalous course of coronary arteries arising from an inappropriate aortic sinus or from another coronary artery. Their technique also provides, I would suggest, an outstanding opportunity to hone our knowledge of cardiac morphology. Thus they argue that their Fig. 2 shows the left coronary artery crossing the "upper part of interventricular septum." In that the interventricular septum separates the cavities of the right and left ventricles, and in that the cavity of the left ventricle is not seen in these sections, it seems to me that their interpretation is an anatomic impossibility. If the artery does, indeed, possess an intramyocardial segment, then surely it must be within the free-standing parietal wall of the right ventricle, which forms the subpulmonary infundibulum.

Robert H. Anderson BSc, MD, FRCPath Department of Paediatrics Imperial College School of Medicine National Heart \& Lung Institute Dovehouse St.

London SW3 6LY, United Kingdom

\section{REFERENCE}

1. Mousseaux E, Hernigou A, Sapoval M, Darmon O, Beyssen B, Gaux J-C. Coronary arteries arising from the contralateral aortic sinus: electron beam computed tomographic demonstration of the initial course of the artery with respect to the aorta and the right ventricular outflow tract. J Thorac Cardiovasc Surg 1996;112:836-40.

12/8/80093

\section{Reply to the Editor:}

We appreciate Anderson's interest in our study ${ }^{1}$ and are happy to clarify the anatomic point concerning the passage of an abnormal left main coronary artery (LMCA) originating from the first segment of the right coronary artery.

In our study, we showed that in patients with the LMCA arising from the right aortic sinus, electron beam com- 